\title{
New hardware, new software, different results: The estimation of left ventricular volumes and ejection fractions with gated myocardial perfusion SPECT
}

\author{
Mark F. Smith, PhD \\ a Department of Diagnostic Radiology \& Nuclear Medicine, University of Maryland School of \\ Medicine, Baltimore, MD
}

Received Nov 2, 2018; accepted Nov 2, 2018

doi: $10.1007 / \mathrm{s} 12350-018-01523-9$

See related article, pp. 1714-1724

It is important to evaluate results from new cardiac imaging technologies in a clinical setting and, when needed, to compare them with those from existing methods. In this issue of the Journal of Nuclear Cardiology, Leva et al. ${ }^{1}$ compare the left ventricular ejection fraction (LVEF), end-diastolic volume (EDV) and endsystolic volume (ESV) derived from a novel myocardial perfusion imaging strategy (IQ-SPECT) with results from a conventional myocardial perfusion imaging strategy employing a low-energy, high-resolution (LEHR) parallel hole collimator and filtered backprojection (FBP) image reconstruction. This novel approach, termed IQ-SPECT by the vendor (Siemens Healthineers, Erlangen, Germany), ${ }^{2}$ has three major hardware and software components. A variable focus collimator $^{3}$ (trade name Smartzoom) has holes that are angled to increase sensitivity in the cardiac region. The orbit of the gamma camera is adjusted to keep the heart in the high-sensitivity part of the field of view of the collimator. Iterative image reconstruction with resolution recovery, i.e., depth-dependent collimator response modeling, is then performed ${ }^{4}$ (trade name Flash3D). For both IQ-SPECT and conventional acquisition and image reconstruction the Quantitative Gated SPECT (QGS; Cedars-Sinai) software package ${ }^{5}$ was used to process the reconstructed gated cardiac perfusion images. The LV

Reprint requests: Mark F. Smith, PhD, Department of Diagnostic Radiology msmith7@umm.edu

J Nucl Cardiol 2020;27:1725-7.

$1071-3581 / \$ 34.00$

Copyright (c) 2018 American Society of Nuclear Cardiology. chamber volumes were determined throughout the cardiac cycle, and the LVEF was computed from the EDV and ESV.

The paper analyzed results from stress myocardial perfusion imaging (MPI) studies performed with Tc$99 \mathrm{~m}$ tetrofosmin. Patients were imaged with long-duration IQ-SPECT acquisition (Method A), short-duration IQ-SPECT acquisition (Method B) and standard clinical acquisition with a parallel hole collimator (Method C). The principal findings were that LVEF values with IQSPECT are biased upward by 3 to $4 \%$ with respect to parallel hole collimator acquisition and that the 95\% limits of agreement ranged between -11 and $+17 \%$. The latter finding is of greater consequence in the clinical environment.

\section{A COMPARISON OF THE METHODS: FACTORS THAT MAY EXPLAIN THE DIFFERENT RESULTS}

When the results of two methods for estimating the same quantities are compared, but there are multiple differences in methodology, it can be very difficult to determine the relative influence of the individual factors contributing to the differences in the results. With a look toward the acquisition and image reconstruction parameters in Table 2 in $^{1}$, one can make an effort to try to understand the possible influence of various factors on EDV, ESV and LVEF. The authors have discussed many of these factors, and I would like to highlight a few of them.

The first major difference, of course, is variable focus collimation for Methods A and B, where the highsensitivity region of the collimator is centered on the heart. The slanted holes of the IQ-SPECT collimator will result in projection raypaths that are not the same as for a parallel hole collimator. In general, they will not be 
perpendicular to the rotation axis of the gamma cameras as is the case for parallel hole collimators. Thus, the effects of attenuation on the projection data will be different, and when image reconstruction is performed without attenuation correction the attenuation artifacts in the images will be different. (The authors indicate that attenuation correction for gated IQ-SPECT is not supported by the vendor.) To be more specific, the ratio of the reconstructed perfusion to the true perfusion will vary spatially within the myocardium due to lack of attenuation correction, and this spatial variation will be different for IQ-SPECT and acquisition with parallel hole collimators. This variation is in addition to other spatial-dependent factors such as wall thickness and differential wall motion and thickening of the myocardium as the heart contracts.

The second major difference is the image reconstruction method, which is iterative with resolution recovery for Methods A and B and FBP for Method C. The noise characteristics of the images will correspondingly differ, with noise remaining more local for iterative reconstruction. The modeling of depth-dependent collimator response in iterative reconstruction will, in principle, improve image resolution if post-reconstruction filtering does not smooth away the features from resolution enhancement. This may affect LV volumes and LVEF, though the authors investigated heart wall thickness as a surrogate for image resolution and found little difference between Methods A and C (Table 5). ${ }^{1}$

The number of projection counts varies between the long (Method A)- and short (Method B)-duration IQSPECT acquisitions, but this had little effect on the ESV, EDV and LVEF bias with respect to the conventional acquisition and processing (Method C) (see Figures 1, 2 and 3 in ${ }^{1}$ )

The number of projections is different for IQSPECT vs conventional acquisition. For IQ-SPECT, there are 17 projections for each of two camera heads for a total of 34 projections over 208 degrees, while for the conventional LEHR parallel hole collimator method there are 34 views from each of two detectors for a total of 68 projections over 180 degrees. It is possible that the different angular sampling could affect the reconstructed images, though there are no obvious angular sampling artifacts (see Figure 5 in ${ }^{1}$ ). Any differences in gamma camera orbits (not discussed in the paper) could also contribute to differences in the reconstructed images.

Ultimately QGS software is used to automatically process all reconstructed gated image sets. This software package determines the endocardial borders and a valve plane near the base of the heart. Various count thresholds are used in the determination of these surfaces, as detailed in Germano et al. ${ }^{5}$ The cardiac surfaces and valve plane location are used in determining the LV chamber volumes at each time gate, and the values at ED and ES are used to compute LVEF. Note that QGS was developed with gated SPECT images that were acquired and reconstructed with standard techniques of that era, typically acquisition with parallel hole collimators and filtered backprojection image reconstruction. The LVEF results from QGS have been extensively compared and validated with results from other modalities and methods. ${ }^{6}$ It is not surprising that there would be some changes in ventricular volumes and LVEF when acquisition and image reconstruction methods are varied. In a recent instructive editorial, ${ }^{7}$ Germano reinforces the notion that repeatability is probably more important than absolute accuracy in a clinical setting.

With the projection data that have been acquired as part of the current study, it may be possible to uncouple some of the image reconstruction effects. For example, the projection data acquired with the parallel hole collimator could be reconstructed with an iterative method with and without modeling of depth-dependent collimator response. One could also study whether the post-reconstruction filter has an influence on QGSderived ventricular volumes and ejections fractions. Realistic analytic and Monte Carlo simulations of IQSPECT acquisition that include modeling of attenuation and depth-dependent collimator response, accompanied by iterative image reconstruction with and without resolution recovery, would be useful to better understand the clinical observations.

\section{DO THE FIGURES TELL THE TALE?}

An examination of Figures 1 and 2 in $^{1}$ reveals some interesting effects. In Figures 1A and 1B, the EDVs for Methods A and B are compared with those of Method C. It is striking that almost all of the volume differences that are below the mean bias line are for mean ventricular chamber sizes less than about $150 \mathrm{~mL}$ while almost all differences are above the mean bias level for mean volumes greater than about $150 \mathrm{~mL}$. Though not as pronounced, a similar effect can be seen in Figure 2 for the ESVs, with biases less than the mean bias tending to be for mean ESVs less than $100 \mathrm{~mL}$. Thus, there may be some dependence of the bias on the LV volume. It would be interesting to examine some of the more extreme differences to try to determine what is responsible for the volume differences. Is it the placement of the valve plane, the endocardial wall boundary, or a combination of both?

A hint of what may be responsible for some of the differences can be seen in Figure $5 c .{ }^{1}$ For this heart and particular time gate, the ratio of the lengths of the walls of the heart to the mid-ventricular diameter are greater 
for IQ-SPECT than for the LEHR collimator method. This may result in greater LV volumes for IQ-SPECT. It could be that IQ-SPECT distorts the shape of the myocardium due to the angulation of the collimator holes and corresponding raypaths during the projection and backprojection steps in iterative image reconstruction. There may also be distortions due to projection data at fewer gamma camera positions. The localization of the valve plane is critically dependent on the decrease in perfusion in the myocardium toward the base of the heart and on the specifics of the algorithm used by QGS to determine the valve plane location. ${ }^{5}$ Even a small change in relative perfusion toward the base of the heart in the reconstructed images could result in significant LV chamber volume changes.

\section{COMPARISON WITH PREVIOUS WORK}

In their Discussion section, the authors comprehensively review studies by other investigators comparing LVEF estimates from gated IQ-SPECT with those from SPECT with parallel hole LEHR acquisition. ${ }^{8-12}$ There are clearly differences among the reported results, though a consensus of the reasons for the differences has yet to emerge. In this context, the current article provides important additional information on whether LVEF results from IQ-SPECT are comparable clinically with those from gated SPECT with LEHR collimation.

\section{CONCLUSIONS}

Clinicians should be cognizant that whenever changes are made to hardware, acquisition or image reconstruction parameters there could be biases or changes with respect to results obtained with previously used parameters and equipment. It is not feasible to develop broad new clinical standards whenever a parameter is changed, and so physicians must rely on their own judgement and local experience when new methods are implemented clinically. There is clearly research yet to be performed that might better explain the reasons for the differences in ventricular volumes and LVEFs between conventional parallel hole collimator methods and the hardware and software methods of IQ-SPECT for gated myocardial perfusion SPECT scans.

\section{Disclosure}

M F. Smith has no conflicts of interest to disclose with respect to this editorial.

\section{References}

1. Leva L, Matheoud R, Sacchetti GM, Carriero A, Brambilla M. Agreement between left ventricular ejection fraction assessed in patients with gated IQ-SPECT and conventional imaging. J Nucl Cardiol 2018.

2. Vija AH, Malmin R, Yahil A, Zeintl J, Bhattacharya M, Rempel $\mathrm{TD}$, et al. A method for improving the efficiency of myocardial perfusion imaging using conventional SPECT and SPECT/CT imaging systems. 2010 IEEE Nuclear Science Symposium and Medical Imaging Conference, 2010;3433-7.

3. Hawman PC, Haines EJ. The cardiofocal collimator: A variablefocus collimator for cardiac SPECT. Phys Med Biol 1994;39:43950.

4. Vija AH, Zeintl J, Chapman JT, Hawman EG, Hornegger J. Development of rapid SPECT acquisition protocol for myocardial perfusion imaging. IEEE Nucl Sci Symp Conf Record 2006;2006:1811-6.

5. Germano G, Kiat H, Kavanagh PB, Moriel M, Mazzanti M, Su HT, et al. Automatic quantification of ejection fraction from gated myocardial perfusion SPECT. J Nucl Med 1995;36:2138-47.

6. Germano G, Kavanagh PB, Slomka PJ, Van Kriekinge SD, Pollard G, Berman DS. Quantitation in gated perfusion SPECT imaging: The Cedars-Sinai approach. J Nucl Cardiol 2007;14:433-54.

7. Germano G. Quantitative measurements of myocardial perfusion and function from SPECT (and PET) studies depend on the method used to perform those measurements. J Nucl Cardiol 2018;25:925-8.

8. Joergensen T, Hansson SH. Evaluation of the left ventricular ejection fraction with gated IQ-SPECT myocardial perfusion imaging. J Nucl Med Technol 2015;43:193-200.

9. Hippelainen E, Makela T, Kaasalainen T, Kaleva E. Ejection fraction in myocardial perfusion imaging assessed with a dynamic phantom: Comparison between IQ-SPECT and LEHR. EJNMMI Phys 2017;4:20.

10. Pirich C, Keinrath P, Barth G, Rendl G, Rettenbacher L, Rodrigues M. Diagnostic accuracy and functional parameters of myocardial perfusion scintigraphy using accelerated cardiac acquisition with IQ SPECT technique in comparison to conventional imaging. Q J Nucl Med Mol Imaging 2017;61:102-7.

11. Yoneyama H, Shibutani T, Konishi T, Mizutani A, Hashimoto R, Onoguchi M, et al. Validation of left ventricular ejection fraction with the IQ*SPECT system in small-heart patients. J Nucl Med Technol 2017;45:201-7.

12. Havel M, Kolacek M, Kaminek M, Dedek V, Kraft O, Sirucek P. Myocardial perfusion imaging parameters: IQ-SPECT and conventional SPET system comparison. Hell J Nucl Med 2014;17:200-3. 